\title{
Pandemia, aprendizajes y tareas por delante para la Educación en Derechos Humanos: evidenciar, evaluar y proponer
}

- Pandemia, aprendizados e tarefas futuras para a Educação em Direitos Humanos: expor, avaliar e propor

- Pandemic, lessons learned and tasks ahead for Human Rights Education: expose, evaluate and propose

Resumen: La autora comparte sus reflexiones sobre la base de lo pasado y vivido durante la pandemia del Covid-19 con la esperanza de que ayuden a las y los educadores a hacerle frente a un futuro incierto y a seguir desarrollando su trabajo educativo crítico y propositivo en el contexto pandémico y, ojalá pronto, post-pandémico. Inicialmente, resume algunos de los aprendizaje que obtuvo de estos duros tiempos respecto a si en la pandemia se incumplen más o menos los derechos humanos y por qué. Después sugiere y analiza las tareas inmediatas que considera que deberían llevar adelante las y los educadores en derechos humanos para contribuir a un mejor entendimiento colectivo y a una lucha más eficaz por proteger los derechos humanos en situaciones de crisis. Las tareas que recomienda realizar se resumen en

1 Doctora en Educación. Consultora educativa del Instituto Interamericano de Derechos Humanos. Investigadora del Informe Estado de la Educación (Costa Rica). anamariarodino@gmail.com 
tres procesos. Primero, evidenciar-o revelar, exhibir, desplegar, o mostrar - las realidades de inequidad e injusticia ocultas o disimuladas en el tejido social que no siempre se ven o se comprenden fácilmente sin un esfuerzo de indagación. Segundo, evaluar, supervisar o monitorear, acción que se refiere a plantear preguntas sobre aspectos a menudo poco examinados sobre el cumplimiento efectivo (o no) de algún derecho, a fin de analizarlos a fondo, encontrar respuestas y divulgarlas. Tercero, proponer, es decir, formular $\neg$ acciones superadoras necesarias, difundir su discusión y recomendar su cumplimiento. Si desde una perspectiva de Educación en Derechos Humanos toda situación de crisis o conflicto representa siempre un reto tanto como una oportunidad, las desventuras que trajo la pandemia del Covid-19 también representan una coyuntura propicia para construir nuevos y potentes aprendizajes sobre los derechos humanos.

Palabras clave: Pandemia Covid-19. Derechos humanos durante la pandemia. Educación en Derechos Humanos

Resumo: A autora comparte suas reflexões sobre a base da experiência vivida durante a pandemia do Covid-19 com a esperança de contribuir com as educadoras e os educadores a fazer frente a um futuro incerto e a seguir desenvolvendo seu trabalho educativo, crítico e propositivo em um contexto pandêmico, e com sorte, pós-pandêmico. Inicialmente resume algumas das aprendizagens que obteve destes tempos duros sobre se durante a pandemia se cumprem mais ou menos os direitos humanos e porque. Na sequência sugere e Analisa as tarefas imediatas que considera que devam levar adiante um aluta mais eficaz para proteger os direitos humanos em situações de crise. As tarefas que recomenda realizar se resume em três processos. O primeiro, evidenciar - ou revelar, exibir, descolar, ou mostrar - a realidade de iniquidades, injustiças ocultas ou dissimuladas em um tecido social que nem sempre sevê meou se compreendem facilmente em um esforço de indignação; Segundo, - avaliar, supervisionar ou monitorar-ações que se proponham a produzir questõ és sobre aspetos pouco examinados sobre o cumprimento efetivo (ou não) de algum direito, afim de analisá-los em profundidade, encontra respostas e divulga-las; Terceiro propor, que dizer, formular, ações necessárias para difundir suas discussões e recomendar seu cumprimento. Se, desde uma perspetiva de Educação em Direitos Humanos toda a situação de crise ou conflito representa sempre uma possibilidade tanto como uma oportunidade, as desventuras advindas da pandemia do Covid-19 também representam uma conjuntura propícia para construir novas e potentes aprendizagens sobre os direitos humanos 
Palavras chave: Covid-19 Pandemia. Direitos humanos durante a pandemia. Educação em direitos humanos

Abstract:The author shares her reflections about the experience of the Covid-19 pandemic with the hope that they can help educators face the uncertain future and continue developing their critical and purposeful work. Initially, the text summarizes some of the lessons learned during these hard times concerning whether human rights are more or less respected during the pandemic and why. Then, it suggests and discusses the immediate tasks that human rights educators should carry out in order to contribute to a better collective understanding and a more efficient effort to protect human rights in situations of crisis. The recommended tasks can be summed up in three processes. First, demonstrate -or reveal, exhibit or display- the realities of inequity and injustice hidden or disguised in the social fabric that are no easily perceived or understood without an inquiry effort. Second, evaluate, scrutinize or monitor, actions referred to posing questions about aspects little examined of the effective observance (or not) of a given human right with the intend of analyzing them in depth, finding answers and divulging them. Third, to propose, that is to suggest necessary restorative actions, make them known and advocate its implementation. Since from an HRE perspective every case of crisis or conflict represents a challenge as much as an opportunity, the hardships of the Covid-19 pandemic also entail a propitious situation to construct new powerful learning about human rights.

Keywords: Covid-19 pandemic. Human Rights during the pandemic. Human Rights Education

\section{Introducción}

En varias ocasiones durante el año 2020, en diálogos a distancia con educadores latinoamericanos en derechos humanos, me referí a los desafíos que enfrenta la Educación en Derechos Humanos (de aquí en adelante, abreviada $\mathrm{EDH}$ ) en tiempos de pandemia. En esta oportunidad quiero compartir algunas nuevas reflexiones sobre la base de lo pasado y lo vivido, con la doble esperanza de que nos preparen mejor para hacerle frente al futuro incierto y nos ayuden a seguir desarrollando un trabajo educativo crítico y a la vez propositivo situado en el contexto pandémico y, ojalá pronto, post-pandémico.

Por un lado, trataré de resumir algunos aprendizajes que obtuve de estos duros tiempos respecto a sien la pandemia se incumplen más o menos los derechos humanos y por qué. Por otro lado, quisiera sugerirlas tareas in- 
mediatas que creo debería llevar adelante la EDH -o, mejor dicho, nosotros, educadores en derechos humanos - para contribuir a un mejor entendimiento colectivo y a una lucha más eficaz por proteger los derechos humanos en situaciones de crisis. Pondré énfasis en el derecho a la educación, pues me es más cercano por mi formación y experiencia, aunque las propuestas pueden aplicarse a todos los derechos. Estas tareas las sintetizo en las acciones de evidenciar, evaluar y proponer.

\section{Aprendizajes}

Repensar los efectos sanitarios, sociales y económicos derivados de la pandemia, ya sea como consecuencia directa de ella o derivados de las medidas de contención que adoptaron las autoridades de cada país, nos permite hacer algunas comprobaciones sobre el respeto los derechos humanos en esta situación de crisis. Consideremos algunas que, por razones de espacio, serán necesariamente breves. ${ }^{2}$

- $\quad$ El Covid-19 en sí mismo, como enfermedad infecciosa, no discrimina. Puede atacar a todas las personas por igual, independientemente de sus atributos individuales. Pero las situaciones particulares en que viven las personas y los colectivos sociales determinan sus mayores o menores posibilidades de contagiarse, la mayor o menor gravedad del contagio y la evolución de la enfermedad. Entre esas situaciones están, por ejemplo: su estado general de salud previa; sus condiciones de vida y de vivienda (espacio y ventilación, existencia de zonas abiertas); los servicios públicos disponibles en su barrio y su hogar(agua corriente, cloacas e instalaciones sanitarias, luz eléctrica), y la calidad de la atención de salud a la que tiene acceso.

En síntesis, las situaciones del contexto de vida son decisivas en el impacto y consecuencias de la enfermedad, en especial de los contextos de mayor vulnerabilidad social.

- $\quad$ Por lo anterior, la pandemia vino a empeorar, a agravar situaciones de inequidad social en materia de acceso a derechos humanos que ya existían desde mucho tiempo atrás. No las creó: las agudizó.

- La pandemia fue utilizada por algunos gobernantes y empresarios inescrupulosos para ocultar o disimular políticas y conductas ilegales, a menudo abiertamente antidemocráticas. Entre ellas el cierre prolongado de órganos de deliberación, como el congreso o los tribunales de justicia, que

2 Pueden profundizarse consultando la Declaración 1/2020 de la Corte Interamericana de Derechos Humanos; la Resolución 1/2020 de la Comisión Interamericana de Derechos Humanos y los numerosos pronunciamiento de organismos internacionales y regionales de derechos humanos que se recogen en el sitio: https://www.corteidh. or.cr/tablas/centro-covid/centro.html. 
impidieron discusiones abiertas al conocimiento público; las compras del Estado realizadas sin cumplir controles administrativo-financieros y de calidad, que facilitaron la corrupción impune, ylas disposiciones extremas de algunos poderes públicos sobre circulación de las personas, que aumentaron arbitrariamente el rigor del distanciamiento social sanitario y restringieron libertades personales.

- Ciertas respuestas de política pública a veces contribuyeron a ampliar brechas sociales e inequidades pre-existentes, como ocurrió con aquellas que no contemplaron una perspectiva de derechos humamos. Por ejemplo: algunas cuarentenas excesivamente dilatadas favorecieron la pérdida de fuentes de trabajo; los seguros o subsidios de desempleo para personas despedidas de sus empleos o con jornadas laborales reducidas a menudo resultaron escasos, breves o inequitativamente distribuidos; los cierres prolongados de escuelas privaron a la niñez de los beneficios psicológicos y sociales de los encuentros presenciales y de servicios adicionales de los centros escolares como alimentación, controles de salud y protección, y las acciones de educación a distancia durante el cierre de escuelas apoyadas exclusiva o mayoritariamente en medios electrónico-digitales excluyeron a amplios sectores del estudiantado que no tenían acceso a los equipos o la conectividad digital indispensable.

- Las respuestas de política pública más eficaces ante crisis como la pandemia del Covid-19 no suelen ser universales y uniformes, sin distinción. En situaciones de inequidad previamente comprobadas deben considerar acciones afirmativas para las poblaciones en condiciones de mayor vulnerabilidad, por ejemplo en materia de trabajo, de calidad de vida, de salud y de educación

\section{Tareas por delante}

Ante estas comprobaciones, sostengo que la EDH está en condiciones óptimas para impulsar acciones -las que, posiblemente por deformación profesional de maestra, yo llamo "tareas"—que contribuyan a comprender y a responder mejor a situaciones de crisis con pleno respeto de los derechos humanos y para corregir o disminuir inequidades preexistentes. Y la EDH puede hacerlo mejor que cualquier otra corriente educativa por dos razones. Una, conceptual y principista: su visión ética holística, la sensibilidad social que se deriva de su perspectiva de derechos y su búsqueda explícita de la justicia social. Otra, pedagógica: su metodología crítica, participativa y profundamente comprometida con la transformación de la realidad.

Las y los educadores en derechos humanos pueden impulsar estas acciones desde su propio espacio de trabajo en instituciones educativas de cualquier nivel, formales o no formales, gubernamentales o civiles. Y pueden 
hacerlo como parte de las actividades que realizan habitualmente como docentes, investigadores o activistas sociales.

¿A qué acciones o tareas me refiero? Frente a la pandemia u cualquier otra situación de crisis, propongo en primer lugar la tarea de evidenciar -que también podemos llamar revelar, exhibir, desplegar, mostrar... ¿ Qué cosa? Las realidades de inequidad e injusticia ocultas, disfrazadas o disimuladas en el tejido de la sociedad, que no siempre se ven a simple vista o se comprenden fácilmente sin un esfuerzo de indagación, de pesquisa.

Pensemos en realidades cotidianas como las siguientes, entre tantas otras:

o Las desigualdades en las condiciones de vida, que no permiten a todos vivir una auténtica vida digna3;

- Las desigualdades en las posibilidades que las personas tienen de ganarse su sustento, que no permiten a todos el disfrute pleno de los derechos laborales y a la seguridad social;

- Las desigualdades entre los servicios de salud pública (gratuita pero generalmente con muchas limitaciones) y los de salud priva da (completa pero solo accesible para quienes pueden pagar sus altos costos), que no permiten a todos recibir la misma calidad de atención de salud;

- Las desigualdades en el acceso a servicios de conectividad y dispo sitivos tecnológicos, que no permiten a todos "participar del progreso científico y los beneficios que de él resulten" (Declaración Universal de Derechos Humanos, Art. 27 ) y, por tanto, el acceso a la educación a distancia digital o virtual, y

- Las violencias dentro de las familias, que golpean a sus miembros en situación más vulnerable: niños, ancianos, mujeres.

Permítaseme aquí un paréntesis para hacer una aclaración metodológica. He citado las realidades anteriores como si se tratara de conclusiones evidentes porque me dirijo a un público de educadores en derechos humanos o educadores en general. Pero trabajando con estudiantes, de cualquier edad o modalidad educativa, esas comprobaciones deben mediarse pedagógicamente de manera que sea ellos mismos quienes lleguen a identificarlas. Si bien el objetivo de este artículo no es discutir estrategias educativas, puedo y debo señalar la mejor posible: la que el gran educador brasileño Paulo Freire llamó la pedagogía de la pregunta (Freire y Faúndez, 2013-1985). En pocas palabras, son las preguntas del educador - NO sus respuestas- las que abren

3 El concepto de vida digna o condiciones de existencia digna fue argumentado por primera vez por la Corte IDH en 1999 en la sentencia del caso "Niños de la Calle (Villagrán Morales y otros) vs. Guatemala" y reiterado en numerosos fallos posteriores. 
nuevos caminos de pensamiento al estudiantado.

Sólo a manera de brevísimo ejemplo, tendremos que trabajar para llegar al reconocimiento de esas desigualdades planteando secuencias de preguntas tales como " $¿ C r e e n$ ustedes que durante la pandemia se afectaron derechos humanos de las personas, de manera total o en parte? ¿Cuáles? Esos derechos, ¿se vieron afectados de igual manera para toda la población? ¿Cómo afectaron a distintos grupos sociales? Fue justa o equitativa esa afectación? Piensen en casos concretos y discutan sus causas y consecuencia".

Volviendo a las propuestas anunciadas al inicio, la segunda tarea que argumento que la EDH debe encarar se expresa en las acciones de evaluar, supervisar e monitorear. ¿Qué quiero decir? Me refiero a plantearse aspectos a menudo poco examinados sobre el cumplimiento efectivo (o no) de algún derecho, a fin de analizarlos a fondo y divulgar sus resultados. En breve, se trata de formular preguntas a las que hay que encontrar respuestas.

Ejemplifico a continuación con problemas por investigar referidos al cumplimiento del derecho a la educación durante la pandemia, algunos provenientes de mi propia experiencia y otros sugeridos por la actual relatora de Naciones Unidas sobre el Derecho a la Educación, Dr. Koumbou Boly Barry, de Burkina Faso, en su informe de Junio de 2020.

- ¿ ¿Cuál fue el impacto real que tuvo el cierre de las escuelas en todos los niveles?

o En el rezago en los aprendizajes infantiles

o En el porcentaje de exclusión (abandono) escolar

o En la alimentación y salud de los niños más pobres

o En el bienestar psicológico y social de la niñez

o En la violencia intrafamiliar contra niños y niñas

- ¿ ¿Cuál fue el resultado efectivo de la educación a distancia digital o vir tual del estudiantado, en todos los niveles?

- Las políticas públicas adoptadas para hacer frente a la pandemia, ¿podrían haber acrecentado desigualdades existentes? ¿Cuáles y cómo?

- ¿Puede la educación a distancia adoptarse como estrategia perma nente para la educación básica obligatoria, es decir, como un nuevo paradigma educativo que reemplace totalmente la interacción social presencial?

- ¿ ¿Son sostenibles los modelos económicos y financieros que sostienen actualmente los sistemas educativos públicos? ¿Cuáles son o pueden ser las consecuencias de una financiación estatal insuficiente de las instituciones educativas públicas?

- ¿ ¿Cuál es el papel que cumplen las empresas o entidades del sector privado en la educación en $\mathrm{X}$ país? La pregunta hace referencia a los casos 
en que este tipo de entidades se involucraron en la educación pública vendiéndole servicios y productos electrónicos para responder a la pandemia, por ejemplo empresas como Facebook, Microsoft, Google, Zoom, Coursera o Huawei, entre otras.

En cuanto a la tercera tarea, creo que es una responsabilidad que la EDH debe asumir: proponer. En otras palabras, formular acciones superadoras necesarias, difundir su discusión y recomendar su cumplimiento. Es decir, una vez que ha identificado problemas de violación de derechos o de agravamiento de violaciones de derechos, ha exhibido sus raíces y evaluado sus efectos, ha de pasar a la acción propositiva. Se trata de concretar sugerencias de políticas públicas o, bien de procedimientos participativos y democráticos para arribar a esas políticas públicas -sean educativas, de salud, o las que correspondan-, elaboradas colaborativamente con especialistas de cada campo, promover su debate público y exhortar a las autoridades a llevarlas adelante. Es un ejercicio obligado de ciudadanía.

Frente a situaciones de inequidad profunda, una posibilidad reconocida y a menudo planteada desde un enfoque de derechos es proponer medidas de acción afirmativa, también llamadas de discriminación positiva, en beneficio de las personas que sufre no han sufrido históricamente mayores violaciones de sus derechos. La acción afirmativa es "una distinción que se realiza con la intención de contrarrestar las desventajas de una persona o un grupo en el acceso o el disfrute de un derecho. (...) debe estar basada en una norma de carácter temporal, dirigirse a un colectivo desfavorecido y buscar prevenir una discriminación negativa o compensar las desventajas que resulten de condiciones físicas, sociales o culturales" (DI BERNARDI et al., 2007, p. 114).En materia de políticas públicas de derechos humanos siempre cabe considerar las de acción afirmativa cuando se intentan revertir patrones estructurales de exclusión. Este tipo de políticas no entran en contradicción con los principios de igualdad y no discriminación porque precisamente su objetivo es avanzar hacia una igualdad efectiva, sumado al hecho que son aprobadas siguiendo procedimientos legales y tienen vigencia por un período fijo, determinado.

A continuación ejemplifico con algunas situaciones comunes durante la pandemia que nos impulsan a pensar propuestas de políticas educativas restauradoras.

- $\quad$ Ante el cierre de escuelas, ¿qué acciones educativas diferenciadas se pueden diseñar según las condiciones de las poblaciones destinatarias con el fin de garantizar una real continuidad del proceso educativo para todo el estudiantado?

- Una posibilidad: Emplear distintos medios para la comunicación edu- 
cativa: no solamente tecnologías avanzadas (educación en línea) sino también tecnologías sencillas (radio y televisión) y medios no tecnológicos (los clásicos materiales impresos).

- ¿Cómo se pueden enmendar o restaurar las insuficiencias que mostró la educación durante la pandemia y los perjuicios que trajo para tantos niños, niñas y adolescentes? Conviene analizar políticas compensatorias como:

o Organizar programas de nivelación para escolares durante vacacio nes o antes de iniciar un nuevo ciclo educativo.

o Suspender el examen o exámenes es finales del bachillerato secun dario.

o Replantear el procedimiento de ingreso a la universidad para los estudiantes secundarios graduados en pandemia.

- ¿ ¿Cómo volver a la escuela lo antes posible sin poner en riesgo la salud de estudiantes y docentes?

- Elaborar colaborativamente propuestas de estrategias que contemplen y equilibren las necesidades educativas tanto como sanitarias

- ¿ ¿Cuál es el procedimiento más justo para tomar decisiones de política educativa que consideren las necesidades de todos los sectores interesados o beneficiarios?

- Explorar la metodología y los procedimientos apropiados que incluyan siempre la participación y discusión directa y democrática con los colectivos afectados.

Desde una perspectiva de EDH, toda situación de crisis o conflicto representa siempre un reto tanto como una oportunidad -la oportunidad de profundizar la sensibilización, la conciencia y el compromiso de acción que nos cabe impulsar como educadores en derechos humanos. Las desventuras que nos trajo la pandemia del Covid-19 representan también una coyuntura propicia para construir nuevos y potentes aprendizajes sobre los derechos humanos.

\section{Referencias}

COMISIÓN INTERAMERICANA DE DERECHOS HUMANOS (2020). Pandemia y Derechos Humanos en las Américas. Resolución 1/2020 del 10 de abril de 2020. Disponible en: https://www.oas.org/es/cidh/decisiones/pdf/Resolucion-1-20es.pdf. Acceso en: 20 jul. 2021.

CORTE INTERAMERICANA DE DERECHOS HUMANOS (2020). COVID-19 y derechos humanos: Los problemas y desafíos deben ser abordados con perspectiva de derechos humanos y respetando las obligaciones internacionales. Declaración 1/2020 del 14 de abril de 2020. Disponible en: https://www. 
corteidh.or.cr/tablas/alerta/comunicado/cp-27-2020.html. Acceso en: 20 jul. 2021.

CORTE INTERAMERICANA DE DERECHOS HUMANOS (2020). Página dentro del sitio web de la Corte IDH con enlaces a Pronunciamientos de Organismos Internacionales sobre el Covid-19. Disponible en: https://www.corteidh.or.cr/ tablas/centro-covid/centro.html. Acceso en: 20 jul. 2021.

CORTE INTERAMERICANA DE DERECHOS HUMANOS (1999). Sentencia en el caso "Niños de la Calle (Villagrán Morales y otros) vs. Guatemala", del 19 de noviembre de 1999, Serie C, nº 63, p. 144-146.

DI BERNARDI, G. F. et al. Derechos Humanos y Ciudadanía. Santillana, Serie Perspectivas. Buenos Aires, Argentina, 2007.

FREIRE, Paulo; FAÚNDEZ, Antonio [1985]. Por una pedagogía de la pregunta. Crítica a una educación basada en respuestas a preguntas inexistentes. Siglo XXI Editores Argentina: Buenos Aires, 2013.

ONU. Preocupaciones, desafíos y oportunidades en relación con los efectos de la crisis de la enfermedad por coronavirus en el derecho a la educación. Informe de la Relatora Especial sobre el derecho a la educación, Dra. Koumbou Boly Barry (Burkina Faso), Junio 2020. (A/HRC/44/39). Disponible en: http://sjc.uva. es/2020/12/15/preocupaciones-desafios-y-oportunidades-en-relacion-conlos-efectos-de-la-crisis-de-la-enfermedad-por-coronavirus-en-el-derecho-ala-educacion/. Acceso en: 20 jul. 202.

ONU. Declaración Universal de Derechos Humanos, aprobada por la Asamblea General el 10 de diciembre de 1948. Disponible en: https://www.un.org/es/ about-us/universal-declaration-of-human-rights. Acceso en: 20 jul. 2021. 